\title{
Carnets
}

Revue électronique d'études françaises de l'APEF

Deuxième série - 11 | 2017

Les écrivains écrivent l'Europe en français

\section{Regards idéographiques sur la France et la langue française dans l'écriture transculturelle de Shan Sa}

\section{Fiorella Di Stefano}

\section{(2) OpenEdition}

1 Journals

\section{Édition électronique}

URL : https://journals.openedition.org/carnets/2333

DOI : 10.4000/carnets.2333

ISSN : 1646-7698

Éditeur

APEF

\section{Référence électronique}

Fiorella Di Stefano, «Regards idéographiques sur la France et la langue française dans l'écriture transculturelle de Shan Sa », Carnets [En ligne], Deuxième série - 11 | 2017, mis en ligne le 30 novembre 2017, consulté le 21 septembre 2021. URL : http://journals.openedition.org/carnets/2333 ; DOI : https://doi.org/10.4000/carnets.2333

Ce document a été généré automatiquement le 21 septembre 2021.

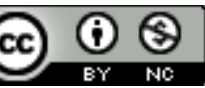

Carnets est mis à disposition selon les termes de la licence Creative Commons - Atribution - Pas d'utilisation commerciale 4.0 International. 


\title{
Regards idéographiques sur la France et la langue française dans l'écriture transculturelle de Shan Sa
}

\author{
Fiorella Di Stefano
}

\section{Introduction et Prémisse méthodologique}

1 Dans les pages de son célèbre essai Orientalisme. L'Orient créé par l'Occident (1978), Edward Saïd propose une image finalement démystifiée des concepts d'«Orient» et d' "Orientalisme », en définissant ceux-ci comme n'étant rien d'autre que des constructions élaborées sous la plume des écrivains ainsi que des journalistes occidentaux. En effet, l'observation du comparatiste :

L'Orient a presque été une invention de l'Europe, depuis l'Antiquité lieu de fantaisie, plein d'êtres exotiques, de souvenirs et de paysages obsédants, d'expériences extraordinaires (...). Pour leur part, les Français et les Anglais - et, dans une moindre mesure, les Allemands, les Russes, les Portugais, les Italiens et les Suisses - possèdent une longue tradition de ce que j'appellerai l'orientalisme, qui est une manière de s'arranger avec l'Orient fondée sur la place particulière que celui-ci tient dans l'expérience de l'Europe occidentale. (Saïd, 2003 : 29-30)

résonne comme une condamnation d'une image de l'Orient longtemps manipulée, voire affectée par le sentiment de supériorité des Occidentaux colonisateurs, voyageurs, touristes, journalistes. En bref, c'est le discours qu'une civilisation tient sur l'autre, comme le suggère Todorov, (idem : 21) en termes de supériorité, d'infériorité et d'unicité, qui a engendré, au fil des siècles, le discours esclavagiste, puis colonialiste, qui comme nous le savons croise à maintes reprises le discours sur l'« Orientalisme ». L'étude de ce dernier dont la présence bénéficie d'une approche pluridisciplinaire, à savoir en politique, en littérature, dans les journaux intimes ainsi que dans la science s'avère, donc, un outil fécond pour connaître moins l'Orient que l'Occident. Pourtant, si le discours orientaliste en Occident a fait l'objet d'importantes études chez les écrivains notamment européens - citons par exemple Marco Polo, Voltaire, Montesquieu, Paul Claudel, 
Malraux, Sartre, est-ce qu'on peut parler d'un discours « européiste » chez les écrivains orientaux qui ont choisi la langue française comme langue d'expression littéraire? Dans ce cas, de quelle manière ce discours se développe et jusqu'à quel point la relation à l'Autre se défini uniquement en termes de supériorité ou plutôt comme appui pour un replacement de la représentation de l'Europe, dans sa version exotique? Ces questions feront l'objet de notre réflexion.

Précisons tout d'abord notre espace géographique d'étude qui sera celui de l'ExtrêmeOrient, à savoir la Chine et de son lien à l'Europe, à savoir la France. En effet notre corpus littéraire inclura les écrivains francophones venus de Chine, qui à partir de la seconde moitié du XXe siècle se sont installés en France et ont commencé à attirer l'attention du lectorat de l'Hexagone, soucieux de lire des histoires de Chine et de Chinois. Notre démarche semble, donc, débuter sur un paradoxe, car les écrivains chinois francophones tels que François Cheng, Dai Sijie, Shan Sa, pour n'en citer que quelques-uns racontent, dans la plupart des cas, des histoires de Chine et de Chinois, qui renvoient donc exclusivement à leur pays d'origine, sans dresser un véritable discours sur l'Europe et les Européens.

4 Qui plus est, dans l'immense réseau des lettres francophones, l'exemple de la francophonie chinoise arrive sur la scène européenne et notamment française au début des années quatre-vingt-dix, au moment où la démystification de l'orientalisme suggérée par Saïd se fait de plus en plus massive, et la Chine toujours racontée par les Européens comme un pays encore trop fermé au monde extérieur, voire immobile, exotique, incompréhensible devient tout d'un coup, à travers la nouvelle génération d'écrivains chinois d'expression française, un espace plus proche, presque familier. Il y a donc deux paradoxes qui se superposent et qui feront l'objet de notre étude, car d'une part les coutumes chinoises décrites dans une langue européenne, à savoir le français, se retrouvent universalisées auprès du lectorat francophone; d'autres part, les coutumes européennes se retrouvent également universalisées à travers les correspondances déclenchées par les figures de style de la langue française superposées aux idéogrammes chinois. C'est donc la manière spéciale de se servir de la langue française, de la travailler, de la manipuler, de la «faire chanter» chez les écrivains chinois-francophones qui nous permettra de replacer l'Europe, à savoir la France, dans sa vocation de « dire le monde $»^{1}$.

5 Notre analyse sur l'écriture, que nous qualifions «franco-chinoise», sera notamment d'ordre sémiotique et rhétorique à la fois. En effet, l'approche sémiotique est étroitement liée aux traits chinois, avec leur surcharge de symboles ainsi que de correspondance tandis que celle de la rhétorique justifie l'emploi des figures de style de la langue française. En effet, nous sommes persuadés que la visualité des idéogrammes superposés aux figures de style fournies par la langue française constitue un lieu privilégié de mobilité, de déplacement tout au long de l'un des sens principaux du corps humain, c'est à dire la vue, car les images de la langue mettent en cause la linéarité de la lecture, en bouleversant les limites entre le centre et la périphérie du texte, et en légitimant l'émergence d'une écriture en mouvement ou plutôt migrante.

C'est le même procédé qui caractérise les enjeux de la mondialisation où l'intensification des échanges d'ordre linguistique, culturel, économique se réalise à travers la mobilité de l'individu qui doit désormais faire face au déplacement physique, psychique et identitaire lié à sa condition de migrant. Il convient de rappeler, à propos du lien entre sémiotique et rhétorique, les études réalisées par le Groupe $\mu$ ou Groupe de Liège, qui à partir des années soixante a élaboré un modèle de rhétorique dont les points forts ont fait l'objet de 
l'étude Rhétorique générale (1970), désormais un point de référence pour les études en sciences humaines. Jean-Marie Klinkenberg, l'un des représentants du Groupe dans sa composition actuelle, s'exprime ainsi lien entre sémiotique et rhétorique :

La rhétorique apparaît ainsi comme une partie créative du système sémiotique: celle qui permet de faire évoluer celui-ci par la production de nouvelles relations entre unités et dès lors par la production de nouvelles unités. Elle est donc un élément moteur, qui se situe en un endroit privilégié : à la frontière, toujours mobile, tracée par les règles du système. Un système, pour rester dynamique, doit en effet toujours comporter un composant évolutif (Klinkenberg, 1996 : 282)

7 Le spécialiste développe les idées déjà amorcées par le Groupe dans le célèbre Traité $d u$ signe visuel. Pour une rhétorique de l'image (1992) où le rapprochement entre les deux disciplines, à savoir la sémiotique et la rhétorique, est analysé dans toutes ses composantes. D'où, notre défi, c'est-à-dire parvenir à une image exotique ou plutôt idéographique de l'Europe à travers la rencontre entre langue française et langue chinoise, dans une dimension transculturelle. Nous nous appuierons notamment sur l'œuvre de Shan Sa, écrivaine appartenant à la nouvelle génération d'artistes venus de Chine, qui dans sa triple carrière d'écrivaine, de calligraphe, et de peintre, nous permet de voyager entre Chine et Europe en stimulant la vue tant dans l'opération de lecture linéaire que de celle de visionnement des images de la langue.

Pour ce faire, tout d'abord nous essayerons de faire le point sur l'actuelle facette exotique de l'Europe qui résulte des relations franco-chinoises, qui au fil des dernières décennies ont enrichi le panorama économique, historique, culturel et linguistique de la France et de l'Europe en général; les études ainsi que les dynamiques sur ce point nous permettront dans un deuxième temps de nous nous atteler à l'analyse de l'œuvre romanesque de Shan Sa, dont le volet biographique ainsi qu'artistique nous fournira un témoignage fécond de sa perception de l'Autre, c'est-à-dire l'Europe, comme lieu à la croisée des langues et des cultures.

\section{Quelques considérations sur la francophonie chinoise comme stratégie du discours « européiste » dans le monde, à travers la langue française : enjeux historiques, culturels et linguistiques}

9 En 2000, les candidats du CAPES et de l'agrégation en France sont confrontés, lors des examens de géographie et d'histoire, à un sujet de débat à la fois inattendu et audacieux, à savoir "La Chine et la diaspora chinoise $»^{2}$. Un signe précurseur ou une simple coïncidence, si on constate que la même année, Gao Xingijan, réfugié politique installé en France depuis 1997 et désormais citoyen français, est couronné par le prix Nobel de littérature, et Dai Sijie, déjà connu auprès du public français grâce à son activité de cinéaste, publie Balzac et la petite tailleuse chinoise, un roman rédigé directement en français, qui fait l'objet, la même année, de plusieurs prix littéraires.

Qui plus est, en 2001 François Cheng, le pionnier de la littérature chinoise francophone au XXe siècle, reçoit le Grand prix de la Francophonie, et en 2002 il est élu Académicien au 34 fauteuil, en donnant ainsi pour la première fois à l'Asie une place d'honneur dans l'illustre compagnie. Shan Sa, quant à elle, remporte le Prix Goncourt en 2000 pour le roman Porte de la paix céleste et le Prix Goncourt pour les Lycéens l'année suivante grâce au 
succès du roman La joueuse de Go. Cette foule (primée) d'écrivains chinois d'expression française semble corroborer la richesse du volet culturel et littéraire de la puissance montante de la Chine envisagée par Alain Peyrefitte dans Quand la Chine s'éveillera... le monde tremblera, en 1973, au lendemain de la visite officielle en Chine de Georges Pompidou. Ensuite les années croisées France / Chine entamées en 2004-2005 semble fortifier du point de vue historique ainsi que diplomatique un lien souvent caractérisé par un état de défiance et d'incompréhensions (vente des armes à Taiwan par les Français lors de la première Guerre du Golfe, incident de la flamme Olympique à Paris, pour n'en citer que quelques-uns).

11 Du point de vue culturel la création des Instituts Confucius en France, et des Alliances françaises en Chine intensifie les échanges des étudiants qui souhaitent apprendre la langue chinoise et la langue française. Selon l'estimation d'Emmanuel Lincot « Le nombre d'étudiants chinois dans notre pays [la France] a dépassé, en 2011, d'après les données officielles de Campus France, la communauté des étudiants marocains (la plus importante jusqu'alors) » (Daniel, Grangé, $2015:$ 177).

12 L'ouvrage collectif dirigé par Sylvester et Thouroude, Traits chinois / Lignes francophones (2012), ou les travaux critiques en 2014 à l'occasion des célébrations pour le 50 anniversaire des relations diplomatiques entre la France et la Chine, à savoir France-Chine. Les échanges culturels et linguistiques: histoire, enjeux, perspectives (2015) soigné par Yves Daniel et d'autres spécialistes, Polyphonies franco-chinoises (2015) sous la direction de Béatrice Bouvier-Lafitte et Yves Loiseau pour n'en citer que quelques, ainsi que le grand nombre de thèses auprès des Universités françaises portant sur la littérature chinoise francophone témoignent sans nul doute de l'intérêt vif, voire de l'engouement de la part des Français à propos de la rencontre entre France et Chine, et bien sûr entre langue française et langue chinoise. Un prisme séduisant, dont les origines remontent au XVII siècle, destiné à renouveler, encore une fois, les notions d'identité francophone, d'écrivain francophone au XXI ${ }^{\mathrm{e}}$ siècle.

13 En effet, si la francophonie, en tant que phénomène littéraire, linguistique, et culturel n'est plus désormais l'exclusivité des 77 pays de ''OIF $^{3}$, nous pouvons affirmer que le cas des écrivains chinois d'expression française pourrait s'inscrire dans le cadre de la francophonie émergente, c'est-à-dire récente, ou plutôt dans celui de la Littérature-monde en français, dont les thèmes, le style, et la compétence plurilingue exercée par les écrivains évoluent sous l'égide de la mondialisation en termes de déplacements, migrations, échanges, suppression de frontières, dans le but finalement d'une réduction des écarts, où se situe d'ailleurs la nature même du concept de convergence littéraire, désormais bien introduit au sein des nouvelles approches des poétiques francophones. Dans l'immense réseau des littératures francophones, qui font de la pluralité linguistique et culturelle leur marque distinctive, il n'est pas anodin que la pratique scripturaire du roman est devenu le terreau privilégié où se reflètent les migrations physiques et identitaires des écrivains FLE ${ }^{4}$.

\section{L'œuvre de Shan Sa ou la manière de calligraphier la langue française et la France}

14 «Coutume importée d'Occident, le Nouvel An ouvre la saison des bals " (Shan Sa, 2001: 47) Ainsi s'exprime la joueuse de Go, protagoniste du roman éponyme de l'un des succès 
français de Shan Sa, créant ainsi un des rares liens directs entre l'Occident, à savoir l'Europe, et la Chine tout au long du roman. La Chine en guerre contre le Japon (1938), comme nous le verrons dans un instant s'ouvre aux traditions venues d'ailleurs. Née à Pékin en 1972, au sein d'une famille d'intellectuels, Shan Sa « bruissement du vent dans la montagne ", son vrai nom étant Yan Ni, en idéogrammes 扇, en pinyin shān, c'est-à-dire "vent ", mais également " montagne », fut poussée depuis son enfance à la calligraphie, tout comme à l'écriture de poème. Son premier recueil en chinois Poèmes de Yan Ni date de 1983. Son adolescence et une première partie de sa phase adulte se déroulent, donc, en Chine, où elle s'affirme comme poétesse, calligraphe, et peintre. Ensuite, en 1989, dégoûtée par les événements de la Place de Tian'an-men, auxquels elle participe comme étudiante, elle décide de quitter définitivement la Chine pour rejoindre son père, Professeur à la Sorbonne, en France. Une fois installée dans l'Hexagone, elle continue ses études à Paris en perfectionnant l'apprentissage de la langue française, en poursuivant sa carrière d'écrivaine entamée en Chine.

Pourtant, cette fois, elle se tourne définitivement vers la langue française, comme langue d'expression littéraire. Ce choix lui permet d'être à l'honneur du marché éditorial français, et par conséquent, du lectorat français qui apprécie ses romans parfumés de Chine comme une sorte de voyage à la recherche des valeurs universelles : «Si l'écrivain fait son livre d'une histoire chinoise, africaine ou autre, les lecteurs y liront les leurs, y vivront leurs souffrances et leurs plaisirs. Dans le roman le monde est universel, l'humanité plus unie. La Chine et la France ne font qu'une (Ding, 1990 : 327).

16 Avec la parution du roman La Joueuse de Go en 2001, Shan Sa parvient sans nul doute à une excellente maîtrise de la langue française ; un apprentissage qui ne s'est pas réalisé sans peine, et qui inscrit l'écrivaine pékinoise dans le cadre des "auteurs FLE», selon la catégorie suggérée par Anne-Rosine Delbart (2005: 145). L'écriture est recherchée et la forme rend hommage à la musicalité de la langue française. L'écrivaine pékinoise, qui ne vise pas à entrer en compétition avec la langue du pays d'accueil, en exploitant, au contraire, toutes le potentialités esthétiques ainsi qu'artistiques, en générale, fournies par la langue française. Par contre, les idéogrammes ne sont jamais utilisés directement dans le texte ; mais leur présence est assurée par le biais des figures de style.

17 Avant de nous plonger dans la lecture ainsi que dans l'analyse de certains extraits de $L a$ Joueuse de Go, dans le but de mettre en évidence la typologie d'alliance entre langue française et langue chinoise menée par Shan Sa, il nous convient d'en rappeler brièvement le dénouement. Parmi les événements historiques qui ont bouleversé les esprits des Chinois, Shan Sa choisit, pour son premier succès en français, celui de la guerre sino-japonaise de 1938, suite à l'occupation de la Manchourie par l'armée japonaise. Dans ce cadre, une lycéenne (nommée "Chant de nuit ", comme elle-même révèlera seulement à la fin de l'histoire), se passionne au jeu du go, soucieuse de vivre un autre destin que celui que l'histoire lui a réservé. Sa vie est bouleversée par l'arrivée d'un officier japonais totalement dévoué à la logique de son Empire. Alors-que la Chine vacille sous les coups de l'envahisseur, la Chinoise et le Japonais s'aiment et s'affrontent en juxtaposant leurs vies à celle du damier du Go.

18 La Joueuse de Go présente, déjà à partir du titre, le procédé stylistique de la métaphore, qu'on pourrait qualifier à double vitesse. En effet, d'une part, la métaphore du Go est exploitée pour célébrer les coutumes et les traditions de l'Empire du Milieu: «Selon la légende, la Chine a inventé ce jeu [le Go] extraordinaire il y a quatre mille ans. Au cours de sa trop longue histoire, sa culture s'est épuisée, et le go a perdu son raffinement, sa 
pureté d'origine » (Sa, 2001 : 153) ; d'autre part le jeu de Go dessine la misère de l'homme (un pion) en guerre, lorsque le jeune officier japonais affirme : « Mon affectation en Chine m'a permis de comprendre la grandeur et la misère du soldat. Conduit par l'ordre, il se déplace en ignorant la direction et le sens de sa marche. Un pion parmi d'autres » (idem : 201). Le jeu du Go comme métaphore de la guerre, et les pions comme le déracinement des soldats et des hommes en générale. C'est au niveau des procédés stylistiques, donc, que le rapprochement entre France et Chine devient de plus en plus évident, surtout dans les modalités d'emploi de la métaphore autour du jeu de Go pour la construction par exemple de la phrase suivante: «cette existence n'est qu'une partie de go » (idem : 275) ou encore de façon moins directe: «Le bonheur est un combat d'encerclement » (idem : 278). En effet, nous pouvons deviner une juxtaposition d'une tradition comme celle du Go, strictement liée à la Chine, mais qui à travers le recours à la métaphore ouvre ses portes et ses « pions » au monde, dans une dimension universelle, et les potentialités de « dire le monde » de la langue française de façon originale sans perturber la syntaxe de cette dernière.

Pourtant, le rapport de Shan Sa à la francophonie s'avère marqué par le sens de l'Histoire de son pays d'origine, par le souci de s'en détacher parfois, mais aussi par le désir poignant d'en louer les beautés. Et les beautés de la Chine sont présentes partout dans La joueuse de Go, notamment dans la description des phénomènes naturels, du paysage, comme nous pouvons le remarquer dans l'incipit: «Place des Mille Vents, les joueurs couverts de givre sont pareils aux bonhommes de neige. Une vapeur blanche s'échappe des nez et des bouches. Des aiguilles de glace, poussées sous le rebord de leurs toques, pointent vers la terre. Le ciel est de nacre, le soleil cramoisi, tombe, tombe. Où se situe le tombeau du soleil ?» (idem : 9).

Et encore : « La lune est absente cette nuit, le vent gémit comme un nouveau-né. Là-haut, un dieu affronte une déesse en bousculant les étoiles » (idem: 10). La similitude entre les " joueurs couverts de givre» et les "bonhommes de neige", les couleurs du ciel, «le tombeau du soleil », et la métaphore du Dieu qui affronte une déesse en bousculant les étoiles nous font savourer la "poésie » de la langue française où se cache moins le désir de transmettre un message que le désir de faire chanter la langue française, à travers les images et surtout à travers le silence, car les joueurs de Go ne parlent jamais, pendant leur partie, comme l'affirme Shan Sa :

Sur la Place des mille vents, le langage est banni et on n'entend que le claquement des pions... J'ai écrit des phrases semblables dans mes autres romans. Dans mon premier roman, Porte de la Paix Céleste, une étudiante chinoise rencontre un adolescent muet. C'est un de mes thèmes favoris : la rencontre de la parole et du silence. L'idée qu'au -delà de l'échange grammatical et intellectuel (...) il y a la sphère du poétique et de l'intuition 5 .

21 En outre, ce qui frappe dans la narration, c'est le manque de détails dans la description des deux protagonistes ; c'est une technique qui laisse beaucoup d'imagination au lecteur, et surtout, malgré le sujet de la guerre sino-japonaise renferme les deux protagonistes tout au long de frontières territoriales bien définies, elle ne parvient pas, toutefois, à canaliser auprès du lectorat français l'appartenance à l'un ou à l'autre espace géographique. Là où l'écrivaine semble parfaitement à l'aise dans la description ponctuelle et exhaustive de certains aspects de la culture japonaise, avec des citations tirées de textes comme Hagakure, elle semble un peu réticente en ce qui concerne les aspects de la culture chinoise. En effet, elle maintient, nous semble-t-il, un semblant hermétique, ou plutôt moins direct à propos de son pays. Cependant, ce qui pourrait se 
configurer comme un paradoxe pour un écrivain qui parle de Chine et qui veut faire connaître "ce qu'est la langue [et la culture] chinoise", n'est rien d'autre qu'une corroboration de son but: la polysémie des idéogrammes engendre avec force un statut d'hermétisme, qui se révèle un caractère essentiel de la culture chinoise. Pourtant il y a des moments dans le roman où l'officier japonais prend parole pour affirmer la supériorité de son peuple sur la Chine, en condamnant le chaos de cette-ci :

Jamais je n'ai connu une ville où les nantis se méfient à ce point de leur richesse tandis que les pauvres luttent désespérément contre la misère. Le désœuvrement de ce peuple confirme mon opinion: L'empire chinois a sombré irréversiblement dans le chaos. Cette vieille civilisation a implosé sous le règne des Mandchous qui refusaient l'ouverture, la science et la modernisation (...). Seuls les Japonais, héritiers d'une culture chinoise pure de tout mélange, ont vocation à libérer du joug européen. Nous rendrons à son people la paix et la dignité. Nous sommes leurs sauveurs. (Sa, $2001: 71$ )

Les figures de style comme outils de création de «cette langue française » ne se limitent pas à la métaphore ou à la similitude, mais elles englobent aussi les assonances, comme dans l'extrait suivant, lorsque la joueuse reçoit une lettre de son cousin Lu: «Ce cousin discret a trempé son pinceau dans l'encre fade. Les idéogrammes en cursive évoluent entre filigranes comme des grues blanches volant dans la brume» (idem: 15). Une remarque s'impose à propos de l'emploi des verbes évoluer et voler; en effet au-delà de l'assonance, il y a de la part de Shan Sa une polysémie qui met sur le même plan l'évolution, à savoir la métamorphose, et le vol comme voyage, comme déplacement, comme détachement de ses racines dans une dimension verticale.

Le respect vers la langue française, dont nous avons parlé auparavant se répercute surtout sur le niveau morphosyntaxique, de sorte que le cadre morphosyntaxique offert par la langue française ne soit pas bouleversé par la présence des mots chinois qui sont pour autant employés de façon discrète et ponctuellement sous forme de pinyin. Comme le suggèrent Rosalynde Sylvester et Guillaume Thouroude, à propos de l'écriture francochinoise de Shan Sa et les autres écrivains venus de Chine : «Leur langue très soignée est (...) une sorte d'hommage à une forme classique» (Sylvester, Thouroude, 2016: 12). L'écrivaine Pékinoise se sert de "cette langue française » sans pour autant parvenir à la délégitimer; au contraire elle cherche à la nourrir en y reproduisant les marques du lettré chinois.

On peut envisager dans l'attitude de Shan Sa, un vrai et propre regard transculturel qui témoigne de la nécessité d'une catégorisation de son parcours et de son œuvre dans l'espace non précisé de la littérature-monde en français. Il faudrait dépasser l'avis de Daniel Delas quand il définit la langue française comme une langue d'adoption : « un lieu de rencontre plutôt que le temple d'une identité permanente ». La condition d'identité multiple demeure solide, par choix, nous le croyons, dans le but de retracer le concept de territorialité de littérature pour répondre au défi de la mondialisation et de l'ouverture des frontières littéraires et géographiques.

\section{Conclusion}

Faute d'un lien conflictuel avec la France, par rapport par exemple aux écrivains beur qui rappellent souvent dans leurs romans l'action colonisatrice de la France, François Cheng, Shan Sa, et les autres semblent témoigner d'une intégration parfaitement réussie. En 
réalité, d'après notre étude, nous pouvons affirmer que la littérature chinoise francophone semble traversée par une double tension due d'une part au processus d'assimilation, car les écrivains chinois francophones apprennent et appréhendent la langue française jusqu'à se conformer à la représentation de la langue française littéraire; et d'autre part, au processus de dissimulation, car les mêmes écrivains font preuve d'un ethnocentrisme lorsqu'ils parlent de la Chine et des personnages chinois.

À ce propos, nous pouvons partager l'intuition de Casanova lorsqu'il affirme qu'il s'agit d'une attitude qui "opère une dépolitisation [déshisoricisation] systématique " (Casanova, 2008 : 217). Shan Sa n'échappe pas à cette ambiguïté, au contraire, elle en fait un cheval de bataille de son œuvre transculturelle. En effet, lorsqu'elle affirme : «j'espère que cette langue française est écrite de telle manière qu'à travers elle on aperçoit ce qu'est la langue chinoise ", elle ne fait rien d'autre que jeter les bases d'un ethnocentrisme et d'un hétérolinguisme à la fois modérés et opérationnels.

En laissant à coté l'engagement souvent obsédant qui est propre, par exemple, de la littérature beur, Shan Sa, poétesse et calligraphe se fait porte-parole de l'engouement du lectorat français envers la Chine et la langue chinoise à travers son propre engouement pour la langue française. Cette dernière, à son tour, fait l'objet d'un double parcours chez Shan Sa, c'est-à-dire celui de l'acquisition comme langue étrangère et celui du FLE au français comme langue d'écriture. Il est donc question à propos de l'œuvre de Shan Sa, mais également de celle des autres écrivains francophones venus de Chine, de problématiser l'adoption de la langue française tant au niveau privé qu'au niveau professionnel.

L'approche rhétorique et sémiotique que nous avons retenue pour l'analyse du plurilinguisme exercé par Shan Sa dans ses romans nous a permis de mettre en évidence le penchant de Shan Sa pour la langue poétique avec ses images, ses assonances, ses descriptions des éléments de la nature, des sentiments comme l'amitié et l'amour. La narration en outre bénéficie de l'alternance entre voix narratives et tableaux sans que les premières soient subordonnées aux secondes ; au contraire, les deux procédés s'appuyant sur un cosmopolitisme particulier sont de plus en plus interchangeables. Le discours d'une France exotique grâce au lien entre langue française et langue chinoise naît sans nul doute de la nature transculturelle et interculturelle de l'écriture choisie par Shan Sa pour dialoguer avec les Européens. Si d'une part elle met en évidence, à travers son roman, sa nature patriotique, d'autre part elle fonde son dialogue sur un tropisme culturaliste tout comme un projet d'universalisation des valeurs, jusqu'à friser l'utopie. Nous sommes loin, bien sûr de pouvoir parler d'un discours " européiste " tout court, comme nous l'avions pensé au début de notre étude, à travers l'œuvre de Shan Sa. Pourtant le corpus littéraire de la francophonie chinoise, comme dans une sorte de damier du Go nous permet de rétablir de façon perpétuelle les enjeux d'une opposition frontale - celle Occident /Orient en termes de dialectique du signe et de la parole. 


\section{BIBLIOGRAPHIE}

ALBERT, Christiane (éd.) (1999). Francophonie et identité culturelles. Paris : Karthala.

AUERBACH, Erich (1946). Mimèsis. La représentation de la réalité dans la littérature occidentale. Paris :

Seuil.

AUGÉ, Marc (2009). Pour une anthropologie de la mobilité. Paris : Payot.

BARTHES, Roland (1975). L'empire des signes. Paris : Seuil.

CASANOVA, Pascale (2008). La République mondiale des lettres. Paris : Seuil.

CROISET, Sophie (2009). Passeurs de langues, de cultures et de frontières : la transidentité de Dai Sijie et Shan Sa, auteurs chinois d'expression française, «TRANS » [En ligne], 81 2009, Paris, Presses Sorbonne Nouvelle, mis en ligne le 08 juillet 2009, [consultée le 28-02-2017]. URL : http:// trans.revues.org/336

DANIEL, Yvan, GRANGE, Philippe et alii (sous la direction de) (2015). France-Chine. Les échanges culturels et linguistiques : histoire, enjeux, perspectives. Rennes : Presses Universitaires de Rennes. DELBART, Anne-Rosine (2005). Les exilés du langage : un siècle d'écrivains venus d'ailleurs (1919-2000). Paris : Presses Universitaires de Limoges.

DETRIE, Muriel (1997). L'écriture chinoise en Occident, dans L. Mabel, M. Hua (éd.), Cultural Dialogue and Misreading. Canberra : Wil Peony.

DETRIE, Muriel (2004). France-Chine. Quand deux monde se rencontrent. Paris : Gallimard. DI STEFANO, Fiorella (2017). «Au de-là des mots français, à travers les traits chinois : le multilinguisme comme migration dans l'écriture franco-chinoise de Shan Sa », JPIC-Journal of Philology and Intercultural Communication, vol. 2, N. 2 p. 7-23.

GAUVIN, Lise (1997). L'écrivain francophone à la croisée des langues. Paris : Karthala. GROUPE $\mu$ (1992). Traité du signe visuel. Pour une rhétorique de l'image. Paris : Seuil. KLINKEBERG, Jean-Marie (1996). Précis de rhétorique générale. Bruxelles : Deboek. SAÏD, Edward (1997). Orientalisme. L'Orient créé par l'Occident. Paris : Seuil. Avec Préface de Tzvetan Todorov.

SALHI, Kamal (2003). Francophone postcolonial cultures : Critical Essays. Laham : Lexinton Books. SHAN, Sa (2001). La Joueuse de Go. Paris : Gallimard.

SYLVESTER, Rosalynde \& THOUROUDE, Guillaume (2012). Traits chinois / Lignes francophones. Montréal : Les Presses de l'Université de Montréal.

TODOROV, Tzvetan (1984). Mikhail Bakhtin: The Dialogical Principle. Trans. Wlad Godzich. Theory and History of Literature, vol. 13. Minneapolis: U of Minnesota Print. 


\section{NOTES}

1. Nous renvoyons au but souhaité par les signataires du Manifeste de la Littérature-monde en français signé en 2007 par quarante-quatre écrivains de langue française.

2. www.deveninrenseignant.gov.fr

3. Organisation International de la Francophonie.

4. Nous reprenons ici la définition que Anne-Rosine Delbart donne à propos des écrivains francophones (2005).

5. http://www.zone-litteraire.com/litterature/interviews/entretien-avec-shan-sa.html

\section{RÉSUMÉS}

La démystification du concept d'« orientalisme ", élaborée par Saïd dans ses célèbres études a sans nul doute fourni de nouvelles pistes pour la relation à l'Autre, au Divers. Aujourd'hui la France et la langue française se situent au cœur du discours sur l'Europe notamment de la part d'écrivains francophones, même quand leurs œuvres portent uniquement sur leur pays d'origine. C'est le cas par exemple du corpus des écrivains venus de Chine comme François Cheng, Dai Sijie, Shan Sa, qui en parlant de Chine et des Chinois semblent élaborer, en filigrane, une nouvelle définition de l'action de l'Europe et de la France Dans notre étude nous souhaitons analyser l'adoption de la langue française de la part de l'écrivaine chinoise francophone Shan Sa, en tant que outil de ( ré) vision ainsi que de ( ré) actualisation de la notion d'Europe, en nous appuyant sur une approche sémiotique et rhétorique de la langue française telle qu'elle est conçue par l'écrivaine pékinoise.

At beginning of XX century the passion for " chinoiserie " throughout French poets has undoubtedly furnish a fake image about Easter lands behind the notion of « Orientalism » forged by Said. Actually, France and French language as country and language of adoption seem to rise up a new idea of Europe in francophone writers and artists. We can mention for example the new literary perspectives including in Chinese-francophone writers such as François Cheng, Dai Sijie, Shan Sa who are very much involved in offering to European readers a new idea of China. However theirs works contributes to develop a new idea of Europe in the world. In this paper we focuses about Chinese- francophone writing as way to light on an "exotic" image of Europe.

\section{INDEX}

Mots-clés : Shan Sa, écriture franco-chinoise, Orientalisme, Transculturalisme, sémiotique

Keywords : Shan Sa, Chinese-francophone writing, transcultural studies, semiotic-Orientalism 


\section{AUTEUR}

\section{FIORELLA DI STEFANO}

Università per Stranieri di Siena (Italie)

distefano@unistrasi.it 